\title{
Não se perca no debate! Mineração de Argumentação em Redes Sociais
}

\author{
João Pedro da Silva Sousa ${ }^{1}$, Rodrigo Costa Uchoa do Nascimento ${ }^{1}$, Renata Mendes \\ de Araujo ${ }^{1,2, \dagger}$, Orlando Bisacchi Coelho ${ }^{1}$
}

\footnotetext{
${ }^{1}$ Faculdade de Computação e Informática - Universidade Presbiteriana Mackenzie ${ }^{2}$ Programa de Pós-Graduação em Sistemas de Informação da EACH/USP. São Paulo, SP, Brasil

Abstract. Argumentation Mining is a subject at the intersection of Computational Linguistics and Data Science that is quite relevant for decision making and includes tasks such as identification and retrieval of the structure of an argument and each participant's utterance polarities. This work is a first step towards establishing an Argumentation Mining process focused on arguments in social networks aimed at recognizing the structure of the debates that occur in the networks. The results of this process are then input to a debate visualization tool, Visu, as a way to foster the understanding of debates between users of social media.

Resumo. Mineração de Argumentação é uma área de pesquisa entre Linguística Computacional e Ciência de Dados, com grande importância para processos de tomada de decisão, possibilitando a identificação e recuperação da estrutura do diálogo e da polaridade dos argumentos usados pelos participantes. Este trabalho é um primeiro passo para construir um processo de análise de discussões em redes sociais com base na Mineração de Argumentação visando compreender a estrutura da discussão que se estabelece nas redes. Os resultados obtidos nesse processo são usados para alimentar a ferramenta de visualização de discussões Visu, de modo a facilitar o acompanhamento e entendimento das discussões em redes sociais.

\section{Introdução}

Vemos, em nossa sociedade, um debate contínuo de valores, conflitos e ideais que refletem, em grande parte, visões individualistas e particulares sobre conceitos, fatos e situações, caracterizando uma tendência de fragmentação e polarização. Isso é observável no discurso intenso que se estabelece e é estimulado pela tecnologia, a Web e as redes sociais. Essas questões têm sido discutidas em estudos relacionados ao discurso sociopolítico e suas implicações [UFRJ 2020].

Apesar do grande acesso aos canais de fala e expressão, nota-se um descompasso entre os atores desta rede - muito se fala, mas será que se dialoga? O que coloca em questão o quanto esse discurso está permitindo que a sociedade debata, compreenda e entenda pontos de vista, principalmente os distintos aos seus. A dinâmica de interação nas redes sociais, usualmente por threads e feeds, ao se tornar intensa e com um número

\footnotetext{
${ }^{\dagger}$ Bolsista DT-CNPq, processo \#313210/2019-5.
} 
grande de usuários, dificulta ou mesmo impossibilita acompanhar a totalidade de uma discussão. Dessa forma, a informação obtida por meio das discussões existentes tende a não ser interpretada por completo pelos usuários.

Em um trabalho de pesquisa anterior, foi desenvolvida uma ferramenta, Visu*, para o estudo do impacto de visualizações para o entendimento de uma discussão a partir de um conjunto de mensagens provenientes de redes sociais [Tavares et. al. 2014]. Uma limitação dessa ferramenta está no processo de extração e análise de dados, já que a inserção do conjunto de mensagens depende de intervenção manual, desde sua obtenção até o processamento responsável por classificar as falas no corpus em análise.

A área de Mineração de Argumentação (MA) é uma área multidisciplinar onde se encontram a Linguística Computacional e a Ciência de Dados, que tem como objetivo identificar, extrair e compreender a estrutura de argumentação em discussões, usando, dentre outras, técnicas de processamento de texto e análise de sentimentos [Lawrence e Reed 2020] [Lytos et.al 2019] [IBM 2020]. Este trabalho surge com o objetivo de compreender as técnicas de MA e explorar sua aplicação em um processo para a análise de discussões em redes sociais seguindo o fluxo (pipeline) proposto por Lippi e Torroni [2016]. A proposta é que os resultados obtidos na execução deste processo possam alimentar a ferramenta Visu com os dados para a visualização da discussão. O processo explorado, e aqui reportado, envolve a extração dos dados das redes sociais - os textos dos diálogos - e uma das etapas fundamentais do processo de Mineração de Argumentação: a classificação da polaridade dos argumentos presentes nesses diálogos.

Este artigo se estrutura como se segue: a Seção 2 apresenta o contexto do problema da pesquisa, o acompanhamento de discussões em redes sociais. A Seção 3 descreve a ferramenta Visu e as visualizações propostas para o entendimento de discussões. A seção 4 apresenta o conceito e objetivos da área de MA. A seção 5 discute trabalhos relacionados. A seção 6 apresenta o processo explorado nesta pesquisa, incluindo as etapas de extração de dados e identificação de posições. A seção 7 conclui o trabalho com as considerações finais, limitações e trabalhos futuros.

\section{Discussões em redes sociais}

Nos últimos anos, é possível notar o papel das redes sociais como forma de construir discussões envolvendo assuntos cotidianos, alguns extremamente polêmicos [Skoric, Zhu, Goh e Pang 2015]. Geralmente, o modo de exibição das falas (e, consequentemente, da discussão) adotado em redes sociais é o feed, que funciona como um mural das notícias mais recentes que se atualiza conforme o usuário navega. Além da organização em feeds, as redes sociais também exibem as manifestações de seus usuários de uma maneira que é possível agrupar comentários e respondê-los diretamente no fluxo da discussão; essa forma de exibição é chamada de thread. Em threads, os comentários ficam com uma estrutura similar à de uma árvore, onde um comentário pode ser um nó com ramificações, ou apenas uma folha isolada.

Contudo, as mídias sociais não foram desenvolvidas especificamente com o objetivo de promover o entendimento do debate e das discussões que acontecem nas redes. Isto porque os mecanismos de feeds e threads não são capazes de identificar relações de argumentação mais complexas entre as falas dos participantes da rede. Há

\footnotetext{
* https://ciberdem.mack.com.br/index.php/ferramentas/
} 
uma contínua cadeia de falas vinculadas à dimensão da temporalidade, muitas vezes sobre temas similares, mas a argumentação - a troca de palavras em controvérsia - tão importante para o entendimento mútuo, é obscurecida.

Compreender a argumentação é importante para que o participante possa se posicionar na discussão, fortalecer ou rever uma opinião e influenciar os demais participantes, visando favorecer a sua visão de solução em uma tomada de decisão ou no debate sobre assuntos cotidianos [Garcia e Klein, 2015]. Identificar essas informações é uma tarefa difícil, pois requer analisar toda a discussão e relacionar as várias mensagens para tentar inferir sobre essas informações que não ficam explicitadas na superfície textual. Quanto mais mensagens trocadas, mais esforço é necessário para compreender a conversação e, consequentemente, acompanhar a discussão.

\section{Visualização de discussões: a ferramenta Visu}

A ferramenta de visualização de discussões Visu [Tavares et. al. 2012] [Tavares et. al. 2014], foi desenvolvida com o objetivo de extrair e apresentar informações de uma discussão em redes sociais, que ajudem a compreender a dinâmica de discussões políticas. Cada informação recebe uma visualização respectiva a suas necessidades: (1) Árvore de Polêmicas: uma estrutura em árvore que apresenta a relação entre os argumentos da discussão mantendo a ordem de acontecimentos e a polaridade de suas relações. Cada nó da árvore representa um argumento e sua coloração representa a polaridade em relação ao anterior. (2) Popularidade: representa o número de referências a um argumento na discussão, considerando a polaridade de suas respostas. (3) Participação: agrupa as manifestações, independentemente de sua natureza, identificando os participantes mais engajados na discussão. Cada "bolha" representa um usuário, e seu tamanho varia de acordo com o número de manifestações. (4) Alianças e Confrontos: agrupa os relacionamentos entre os usuários, levando em conta as manifestações entre eles. Cada nó representa um usuário, e as linhas, a relação de aliança ou confronto entre os usuários de acordo com as cores (verde/vermelho).

Experimentos realizados com a ferramenta [Tavares et. al. 2014] concluíram que as visualizações propostas, quando comparadas à forma típica de discussão em mídias sociais (especificamente, o Facebook), possibilitam uma identificação mais acurada e rápida das informações de interesse definidas, com impactos no entendimento mútuo e tomada de decisões. No entanto, a forma de extração de informações das mídias para a ferramenta é realizada de forma manual, inviabilizando as possibilidades de compreender discussões acontecendo nas redes de forma contínua e dinâmica.

As visualizações na ferramenta são apresentadas a partir de estrutura de dados de entrada que seguem modelos típicos de estruturação de discussão [Burgess Yakemovic e Conklin 1990], e contém: id (identificador da mensagem), remetente (nome do participante que enviou a mensagem), data (data de envio da mensagem), horário (horário de envio da mensagem), conteúdo (conteúdo da mensagem), tópico (tópicos os quais a mensagem está inserida), posição (posição adotada na mensagem, que pode ser de apoio, crítica ou neutra) e destinatário (id da mensagem destinatária).

\section{Mineração de Argumentação}

Argumentação é o processo pelo qual argumentos são construídos, compartilhados e avaliados a partir de outros argumentos [Palau e Moens 2009]. Uma argumentação se 
estrutura a partir de evidências, premissas, fatos e falas que suportam ou não uma determinada alegação, em uma cadeia de raciocínio que leva à conclusão de discussões e à tomada de decisão. A argumentação tem papel importante nas atividades humanas, tanto sociais, políticas, jurídicas, científicas como de negócios, e tem sido compreendida como uma área de pesquisa que surge com base em campos como a Retórica e a Filosofia, mas que hoje inclui estudos de Processamento de Linguagem Natural, modelos teóricos de discussão e de recuperação de informações [Palau e Moens 2009] [Lawrence et. al. 2012] [IBM 2020]. O objetivo da MA é detectar a estrutura de argumentação de um documento ou de uma base de discussões. Ela tem como áreas antecessoras a Mineração de Opiniões e a Análise de Sentimentos, entre outras [Lawrence e Reed 2020] [Lytos et.al. 2019].

As questões principais que envolvem a Mineração de Argumentação incluem [Palau e Moens 2009] [Lawrence e Reed 2020]: i) a existência e a qualidade do corpus de treinamento e testes; e ii) as etapas do processo de mineração. Pelo fato de ser uma área ainda recente, ainda não há um conjunto de datasets ideal, datasets (corpora, já que seriam datasets textuais) que possam ser usados para comparações do desempenho de ferramentas distintas, embora haja esforços da comunidade no sentido de gerá-los.

Em linhas gerais, o processo de MA pode ser visto com um pipeline que leva de documentos textuais não estruturados a um argumento estruturado, que pode ser representado como um grafo de argumento, onde os nós são os argumentos e as relações entre os argumentos são as arestas [Lippi e Torroni 2016]. Esse pipeline é composto por duas macro-etapas: i) a detecção da sentença argumentativa, isto é, dos trechos de um dado corpus onde residem os argumentos, e suas respectivas fronteiras, e ii) a previsão da estrutura global da argumentação, as relações entre os vários argumentos usados ao longo de uma argumentação. A primeira macro-tarefa, a detecção da sentença argumentativa, por sua vez, se caracteriza por duas tarefas: i) a extração das sentenças argumentativas e ii) a detecção das fronteiras entre essas sentenças argumentativas. Uma das tarefas essenciais na extração das sentenças argumentativas é a identificação da polaridade, ou análise de sentimentos, das sentenças que efetivamente são argumentativas, exatamente o foco do trabalho aqui reportado.

Lytos e colegas [2019] instanciam o referencial desenvolvido por Lippi e Torroni [2016] para a mineração de argumentação em redes sociais, propondo um referencial em quatro passos, que consiste em: i) detecção de argumento; ii) identificação das relações, isto é, a previsão da estrutura do argumento; iii) detecção de postura (stance) e iv) tarefas relacionadas a confiabilidade. Detecção de argumento corresponde à detecção da sentença argumentativa, no referencial de Lippi e Torroni. O segundo passo, a identificação das relações, consiste na identificação das eventuais relações de apoio, ataque ou neutras expressas em cada uma das sentenças argumentativas previamente identificadas. Já a detecção de postura se propõe a caracterizar a estrutura global da argumentação, bem como se a argumentação, em sua totalidade, é favorável, contrária ou neutra em relação a seu tópico. Por fim, o quarto passo funciona como uma etapa opcional onde se encontram tarefas como detecção de rumor, marketing viral e detecção de fake news, entre outras. Apesar de técnicas de análise de sentimento serem utilizáveis nos passos (ii) e (iii) do referencial, o trabalho aqui reportado está focado no passo (ii) identificação de relações. Essas etapas desafiam, em grau crescente de complexidade, as técnicas atuais de Processamento de Linguagem Natural, ao ponto de provocar o surgimento de uma comunidade de pesquisa específica ao tema [ArgMining 2021]. 


\subsection{Desafios de Mineração de Argumentação em redes sociais}

As tarefas de mineração de argumentação são impactadas pelo grau de coesão e do texto sendo analisado [Lytos et. al. 2019]. O cenário em redes sociais foge dos padrões esperados, criando alguns desafios para o processo de análise e validação.

O primeiro desafio é a maneira que as informações estão dispostas na rede informações fragmentadas que se perdem com o tempo. $\mathrm{O}$ uso de threads favorece o entendimento de apenas uma das várias linhas de pensamento sendo discutidas (iniciadas pelo autor da thread), o que torna difícil conectar diferentes linhas de argumentos para formação de uma discussão. Além disso, é comum a presença de falas isoladas no discurso, ou seja, apesar de agregarem para a discussão com um comentário, eles não se conectam com nenhum outro argumento prévio, como se seus autores estivessem falando sozinhos. Para a solução deste desafio, é necessário organizar os dados disponíveis usando alguma estratégia que seja capaz de conectar o diálogo. Uma abordagem simples e eficaz é escolher uma rede social em que os usuários naturalmente debatem de forma mais organizada, por exemplo, a rede social Reddit, onde os usuários tendem a argumentar sobre determinados tópicos de maneira mais próxima de um debate do que no Twitter, que apesar de possuir um volume maior de manifestações, geralmente estas não estão relacionadas diretamente [Abbott et. al. 2016].

O segundo desafio surge devido à informalidade da linguagem utilizada nas redes sociais. Enquanto as estratégias iniciais da MA trabalhavam com textos formais, como sentenças de tribunais ou projetos de lei [Lytos et. al. 2019], a problemática atual está na maneira como a discussão é feita, com presença de gírias, abreviações, diversos erros gramaticais etc. As estratégias usadas para contornar a linguagem informal no texto são uma adaptação do processo de normalização de texto, já utilizado frequentemente em MA. Dessa forma, algumas gírias ou variações linguísticas são reduzidas a palavras mais adequadas ao dicionário da língua. Entretanto, devido a esse processamento de texto, em alguns casos é possível haver perda de informação por alterar o significado da frase dentro do contexto de uma discussão.

Uma vez contornados os desafios, abre-se o caminho para análise de polaridade dos argumentos da discussão identificada. Existem algumas formas de abordar a etapa de classificação de discussões em um processo de MA; entre elas está o processo de análise de sentimento [Paltoglou e Thelwall 2012] [Zhai e Massung 2016] [Agarwal et. al. 2011], utilizado neste trabalho.

\section{Trabalhos Relacionados}

O desafio de acompanhar e entender discussões no cenário de interações das redes sociais é observado no trabalho de Rodrigues et al. [2013] que, utilizando a rede social Twitter, constroem uma técnica de identificação de sentimentos baseada nas postagens da rede em que, com o uso de tokenização e expressões regulares, medem qual o sentimento sobre determinado tópico com base na quantidade de sentimentos positivos ou negativos identificados nas postagens. O trabalho de Nascimento et. al. [2020] complementa a técnica de análise de sentimentos com o uso de uma ferramenta capaz de extrair os valores lexicais das palavras, com a mesma finalidade de identificar sentimentos em argumentos. O trabalho de Dusmanu et. al. [2017] apresenta métodos de detecção de argumentos nas discussões, usando um modelo de argumento proposto por Toulmin [2003], na rede social Twitter. O escopo do trabalho se resume à tarefa de classificar os argumentos de um 
conjunto como informativos ou não-informativos, ao, por exemplo, diferenciar argumentos que contenham links externos, imagens ou informações não relacionadas aos argumentos que contribuem para a discussão.

No geral, ferramentas de mineração de argumentação visam entender a argumentação, definir uma estrutura de um argumento ou classificar aqueles relevantes para a discussão. Nosso diferencial é usar técnicas de Análise de Sentimentos baseadas em Aprendizagem de Máquina para compreender o posicionamento de cada falante sobre o tema abordado, através das polaridades das falas e como as outras falas dos participantes da discussão são influenciadas entre si.

\section{Explorando um Processo de Detecção de Argumentos}

Exploramos o pipeline definido por Lippi e Torroni (2016) de forma a obter os dados requeridos pela ferramenta Visu (falas, emissor-receptor das falas e polaridade das falas). Desta forma, para a etapa de detecção das sentenças argumentativas, previstas no pipeline, consideramos como sentenças cada uma das falas dos participantes na discussão. Para cada uma delas, utilizamos técnicas de análise de sentimentos para determinar sua polaridade. Foram utilizadas técnicas de análise de sentimento para definir a polaridade das manifestações no discurso, utilizando o otimizador SGD [Pedregosa et al. 2011] para a classificação, treinado com as discussões já classificadas presentes na base IAC.

Em relação à etapa de previsão da estrutura global da argumentação, definida pelo pipeline, o processo compreendeu a identificação simples do direcionamento das falas entre os participantes, conforme requerido pela Visu. Foram utilizados os identificadores extraídos junto com os textos das redes sociais, de modo a criar uma estrutura de dados capaz de apontar a relação entre um argumento e outro na discussão, mantendo a estrutura de threads da discussão.

A base utilizada para o experimento foi a Internet Argument Corpus (IAC) [Walker et.al. 2012], contendo discussões com um teor politizado, porém amplas o bastante para que pudéssemos usá-la em debates mais genéricos.

O ferramental desenvolvido para apoio ao processo está disponível em: https://github.com/ciberdem/MineracaoDiscussoesRedesSociais A pesquisa segue uma metodologia experimental (Figura 1), cujos passos são descritos nas seções a seguir.

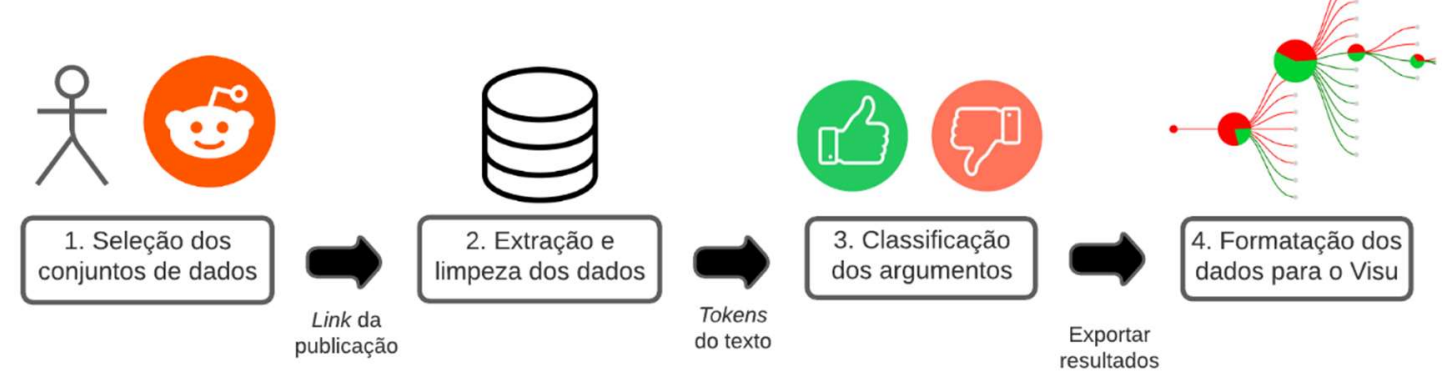

Figura 1. Processo experimental para mineração de argumentação em redes sociais.

\subsection{Seleção de bases}

O desafio da etapa de seleção consiste em encontrar conjuntos de dados que envolvam efetivamente o contexto de uma discussão, para uso nos processos de treinamento e testes 
dos modelos e aplicação em um contexto real. Um aspecto importante aqui, foi a escolha de uma base que estivesse anotada com a polaridade de cada posicionamento emitido, indicando se argumentos tinham um teor positivo, negativo ou neutro. Além disso, existe uma escassez de bases de discussões anotadas e contextualizadas com a proposta deste projeto, nos limitando a usar bases extraídas do IMDb e posteriormente, do IAC. Dessa forma, ganhou-se tempo de desenvolvimento, pois a base pôde definir um contexto para as discussões analisadas, bem como remover a necessidade de anotar os argumentos manualmente para base de treinamento para as etapas seguintes.

Inicialmente foi usada uma base rotulada do site IMDb [Maas et.al 2011], contendo avaliações dos usuários sobre diversos filmes, bem como o teor da crítica, indicado como positiva ou negativa. Apesar de ser uma base satisfatória, ela não atendia completamente às necessidades do trabalho, pois para desempenho e métricas satisfatórias, era preciso uma base com foco exclusivamente em discussões. Ao final do projeto, a função da base IMDb serviu apenas como métrica de comparação de resultados dado que não haviam outras bases para realizar tal comparação.

Dentre algumas outras bases encontradas, a que mais atendia os requisitos do trabalho era a IAC [Walker et. al. 2012], composta exclusivamente por discussões de variados assuntos retirados de fóruns de redes sociais, com a presença de rótulos condizentes com a polaridade esperada dos argumentos. Assim, a base IAC compôs os conjuntos de treinamento e teste.

\subsection{Extração e limpeza dos dados}

Para extração dos dados, a ferramenta de extração (scraper) foi construída utilizando a linguagem de programação Python, em conjunto com uma API da rede social Reddit através da biblioteca PRAW [Boe e Payne 2016]. A partir de um link para um post de discussões no Reddit, a ferramenta extrai os comentários das postagens mantendo a estrutura de relação entre eles, como, por exemplo: autor, data e hora, conteúdo do texto, referências e citações a outros usuários ou seus comentários. O scraper mantém a estrutura de discussão dentro do modelo lógico utilizado pela ferramenta Visu.

Para utilização dos dados, tanto os extraídos via scraper construído, quanto os obtidos pela base IAC, foi necessário usar uma etapa de pré-processamento, para remover algumas características indesejadas que podem impactar o resultado das classificações. Para a base IAC, foi necessário remover do texto elementos como links, caracteres especiais e elementos de formatação do texto. Quanto às bases extraídas do Reddit via scraper, foi preciso remover comentários vazios espalhados no meio dos argumentos, devido a uma característica da rede social que permite exclusão futura de comentários quando os usuários são banidos da plataforma. Quando removidos da discussão, seus argumentos têm o texto oculto, porém, mantêm algumas informações dos usuários que o publicaram.

\subsection{Classificação dos argumentos}

Quanto ao método de classificação dos argumentos, de acordo com os requisitos da ferramenta Visu, a classificação se deu sentença a sentença, fornecendo três rótulos que caracterizavam a polaridade de um argumento: apoio, oposição ou neutro. A primeira alternativa envolvia uma biblioteca do Python chamada NLTK [2021], que se encarrega da etapa de classificação. Entretanto, a falta de customização de parâmetros e a 
impossibilidade de treinamento dos modelos usados para a classificação descartaram a alternativa. Apesar da biblioteca apresentar um bom desempenho para classificações de frases simples, as barreiras apresentadas não permitem o uso desse mesmo método para classificar argumentos de uma discussão com a mesma acurácia.

A solução escolhida para o classificador foi o SVM, com base no otimizador SGD implementado pela biblioteca Scikit-learn [Pedregosa et. al. 2011], usando métodos de classificação linear. SGD é um classificador linear muito usado em problemas de processamento de linguagem natural e classificação de textos, principalmente pela sua eficiência e facilidade de lidar com bases de dados de grande porte. Por meio da exploração automática dos hiperparâmetros, foi possível encontrar parâmetros para o modelo de aprendizado de máquina que configuram uma boa solução, mantendo o equilíbrio entre o ajuste ao conjunto de treinamento e a capacidade de generalização.

Para treinar o modelo do SGD, usando como conjunto de dados a base IAC, a entrada passa por quatro tipos de processos de Text Vectorization, e o processo com maior assertividade na pontuação de validação é escolhido para o Hyperparameter Tuning. Com os parâmetros mais otimizados definidos, é possível carregar e treinar o modelo de SGD para a classificação dos argumentos extraídos via scraper. Os resultados da classificação são finalmente exportados para um arquivo alimentando a ferramenta Visu.

\subsubsection{Assertividade das classificações}

Como resultados iniciais, o algoritmo classificador SGD utilizou como conjunto de dados a base do site IMDb, obtendo uma assertividade de 90\% com os conjuntos de teste do mesmo contexto, como observado em sua matriz de confusão (Figura 2a). Entretanto, dois problemas dificultaram o uso da base IMDb como a principal base de treinamento para as classificações: os rótulos de classificação presentes na base IMDb possuem apenas polaridades de oposição e apoio, assim não conseguindo representar um posicionamento real dos participantes.

Utilizando a base IAC como dataset de treinamento, ao redefinir os parâmetros fornecidos pelo Hyperparameter Tuning, a precisão inicial obtida foi de 70\%, e após o balanceamento correto dos dados rotulados, a precisão do modelo subiu para $74,3 \%$ usando os conjuntos de testes fornecidos. Os parâmetros finais, definidos pelo tuning, foram: modelo linear de classificação modified_huber e taxa de aprendizado adaptive.

Com os três rótulos para a classificação de polaridade dos argumentos (apoio, oposição e neutro) introduzidos pela base IAC, é possível obter uma fidelidade maior dos posicionamentos dos participantes das discussões analisadas. Observando a matriz de confusão dos resultados obtidos (Figura 2b), os pontos que mais diminuem a precisão do algoritmo estão entre as classificações de oposição e neutras. Enquanto argumentos de apoio são classificados corretamente na maioria das vezes, argumentos neutros ou de oposição têm uma linha tênue de separação no modelo linear do algoritmo. 


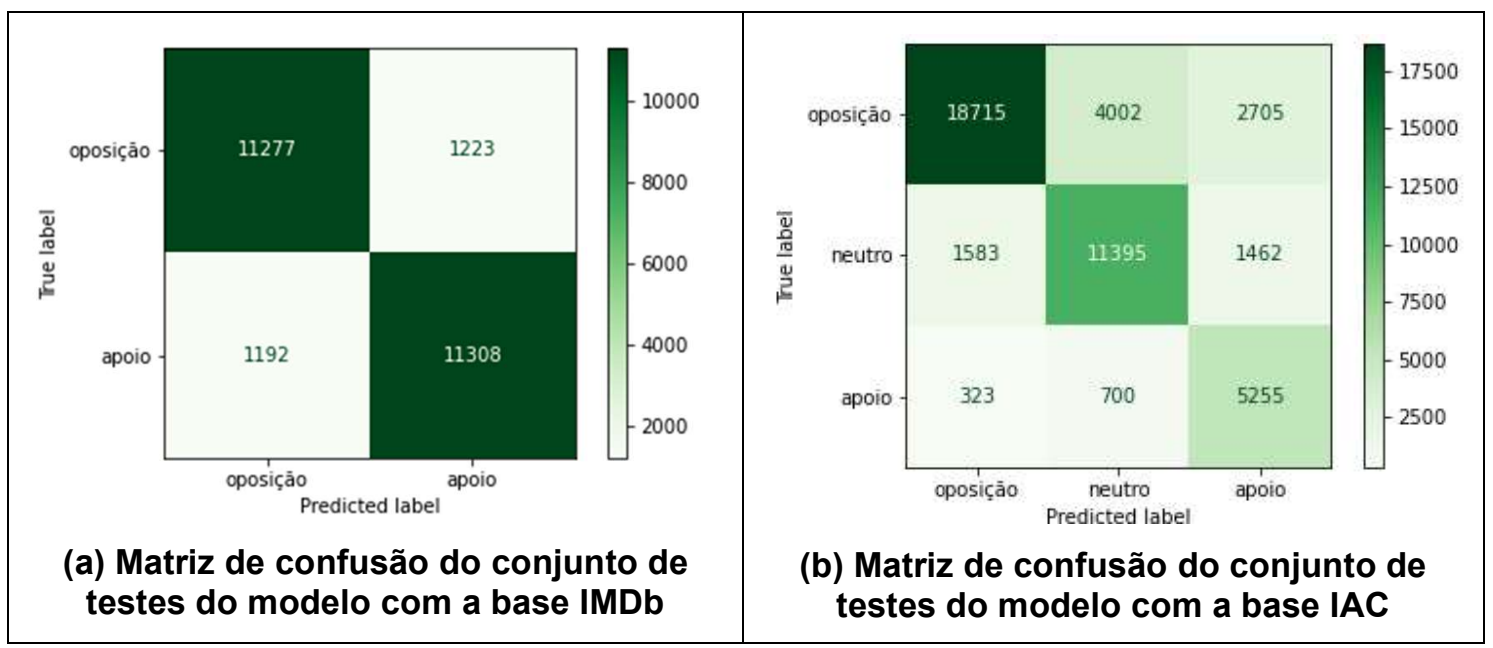

Figura 2. Matrizes de confusão do conjunto de testes do modelo.

Quando comparamos o desempenho de ambos os modelos para classificar as extrações do Reddit, cuja discussão aborda o adiamento das eleições dos Estados Unidos em 2020, observando uma amostra desta classificação (Tabela 1), a adição da polaridade "neutra" é a principal diferença entre os resultados das classificações; em alguns casos introduzindo classificações diametralmente opostas. Apesar da métrica de precisão obtida com a base IAC ser menor que a obtida pela base IMDb, os resultados de classificação da base IAC, em um cenário real, são mais versáteis e fidedignos para análise dos argumentos, pois não necessariamente todo argumento se posiciona sobre um assunto.

Tabela 1. Comparação das classificações realizadas pelo algoritmo treinado pelas bases IAC e IMDb.

\begin{tabular}{|c|c|c|c|}
\hline \multirow{2}{*}{ Autor } & Argumento & \multicolumn{2}{c|}{ Classificação Obtida } \\
\cline { 3 - 4 } & Same here in Washington. O PNW & IMDb & IAC \\
\hline zimmertr & Bet you never looked at it that way! & Apoio & Neutro \\
\hline Veldron & $\begin{array}{c}\text { Ah ok that makes more sense. I misunderstood and thought } \\
\text { you were arguing the perspective that since the 2A appears } \\
\text { questionable in it's ability to prevent tyranny, that it } \\
\text { Shouldn't exist... Which opens up a whole other can } \mathrm{f} \\
\text { worms (natural rights to self defense etc etc) }\end{array}$ & Oposição & Apoio \\
\hline
\end{tabular}

\subsection{Visualização dos dados na ferramenta Visu}

Os resultados provenientes da classificação dos argumentos precisam de uma formatação específica para serem aceitos pela ferramenta Visu. O arquivo contém os seguintes dados para o processamento (Tabela 2): identificador, autor, data, hora, mensagem, assunto, polaridade e destinatário. Os dados são resultado do processo de mineração de argumentação e análise de sentimentos, gerando uma tabela que é formatada para texto bruto, indentado por espaçamentos e tabulações. 
Tabela 2. Dados exportados para a ferramenta Visu.

\begin{tabular}{|c|c|c|c|c|c|c|}
\hline Id & Autor & Data & Argumento & Assunto & Polaridade & Destino \\
\hline 3075 & Thousand_Eyes & $\begin{array}{c}30 / 07 / 20 \\
12: 55\end{array}$ & $\begin{array}{c}\text { my understanding is he's a middle ground in } \\
\text { terms of dems, not amazing or super left } \\
\text { leaning but solidly in the middle. }\end{array}$ & $\begin{array}{c}\text { Donald Trump calls } \\
\text { for delay to } 2020 \\
\text { US presidential } \\
\text { election }\end{array}$ & positive & 2759 \\
\hline 3076 & wtfcanunot & $\begin{array}{c}30 / 07 / 20 \\
12: 23\end{array}$ & $\begin{array}{c}\text { I feel like if this happens, it will be the same } \\
\text { thing happened to Melania. I just wanted to } \\
\text { be trophy wife, did not want to be First } \\
\text { Lonald Trump calls } \\
\text { for delay to 2020 } \\
\text { US presidential } \\
\text { election }\end{array}$ & negative & 2759 \\
\hline 3077 & IThinkThings & $31 / 07 / 20$ \\
$00: 24$ & $\begin{array}{c}\text { Pro Tempore. Assuming Biden picks a sitting } \\
\text { Senator as VP, they likely just choose that } \\
\text { person as Pro Tempore. }\end{array}$ & $\begin{array}{c}\text { US presidential } \\
\text { election }\end{array}$ & negative & 2759 \\
\hline
\end{tabular}

\section{Conclusão}

Com o desenvolvimento deste trabalho, exploramos uma primeira visão de um pipeline capaz de extrair e classificar argumentos de discussões de redes sociais, para alimentar a ferramenta de visualização de dados de discussões Visu. Os resultados obtidos demonstram uma precisão de $74 \%$ na identificação das polaridades dos argumentos, criando assim uma alternativa confiável à classificação manual feita para alimentar a ferramenta de visualização pelos criadores, atingindo os objetivos propostos. Porém, o processo possui algumas limitações que podem ser aperfeiçoadas em trabalhos futuros.

A precisão das classificações pode ser incrementada se utilizada com um conjunto de dados de discussão maior. As maiores bases de discussões, fornecidas pelo projeto IAC [Walker et. al. 2012], não foram utilizadas por limitações de hardware para processamento. A matriz de confusão obtida com as classificações do algoritmo SGD, treinado pela base IAC, também indica limitações do modelo obtido quanto às fronteiras de separação entre as classes. Em particular, há um grau de confusão entre argumentos com polaridades de oposição e neutras maior do que o desejado, prejudicando a precisão da classificação para estas polaridades. Além disso, uma análise qualitativa pode ser feita a fim de compreender quais estruturas linguísticas não estão sendo identificadas.

Trabalhos futuros podem explorar o método de classificação por regressão logística do classificador SGD. Durante o desenvolvimento, o modelo apenas recebeu parâmetros de configuração para modelos de classificação lineares. Além disso, um hardware mais potente seria mais apropriado para que o treinamento das bases seja menos oneroso. Um trabalho derivado do presente trabalho, e já em desenvolvimento, concebido como um aprimoramento experimental deste, consiste na aplicação de técnicas de deep learning para aperfeiçoar a classificação de polaridade dos argumentos.

Quanto às visualizações obtidas pelo Visu, é necessário realizar ajustes quanto à capacidade de exibição gráfica para um volume maior de dados. $\mathrm{O}$ foco da ferramenta foi um conjunto pequeno de argumentos, então os gráficos exibidos não são escaláveis. Assim, apesar de conseguir gerar gráficos com um número elevado de argumentos, a visualização dos dados fica prejudicada por problemas de renderização e resolução.

As soluções desenvolvidas abrem espaço para estudos posteriores na área de MA, além de possibilitar a popularização deste e de outros campos de Ciência de Dados para o público geral. De fato, pelo contexto inserido no trabalho envolver redes sociais, surgem 
espaços de comunicação com o público que podem despertar o interesse para este e outros cenários de conhecimento.

\section{Referências}

Abbott, R., Ecker, B., Anand, P. e Walker, M. (2016) "Internet argument corpus 2.0: An sql schema for dialogic social media and the corpora to go with it." In Proceedings of the Tenth International Conference on Language Resources and Evaluation (LREC'16) (pp. 4445-4452), https://www.aclweb.org/anthology/L16-1704/

Agarwal, A., Xie, B., Vovsha, I., Rambow, O. e Passonneau, R. (2011) "Sentiment analysis of twitter data." In Proceedings of the Workshop on Languages in Social Media, LSM'11, https://dl.acm.org/doi/10.5555/2021109.2021114

ArgMining (2021) “The 8th Workshop on Argumentation Mining", https://2021.argmining.org/

Boe, B. e Payne, J. (2016) "PRAW: The Python Reddit API Wrapper" GitHub. https://github.com/praw-dev/praw

Burgess Yakemovic, K. C. e Conklin, E. J. (1990) "Report on a development project use of an issue-based information system." In Proceedings of the 1990 ACM conference on Computer-supported cooperative work (pp. 105-118), https://doi.org/10.1145/99332.99347

Dusmanu, M., Cabrio, E. e Villata, S. (2017) “Argument mining on Twitter: Arguments, facts and sources." In Proceedings of the 2017 Conference on Empirical Methods in Natural Language Processing (pp. 2317-2322), http://dx.doi.org/10.18653/v1/D171245

Garcia, A. C. e Klein, M. (2015) "Making sense of large-group discussion using automatically generated RST-based explanations". Available at SSRN 2554838, http://dx.doi.org/10.2139/ssrn.2554838

IBM (2020) "Project Debater", https://www.research.ibm.com/artificialintelligence/project-debater/index.html

Lawrence, J., Bex, F., Reed, C. e Snaith, M. (2012) “AIFdb: Infrastructure for the Argument Web.” In COMMA (pp. 515-516), https://doi.org/10.3233/978-1-61499111-3-515.

Lawrence, J. e Reed, C. (2020) "Argument mining: A survey." Computational Linguistics, 45(4), 765-818, https://doi.org/10.1162/coli_a_00364

Lippi, M. e Torroni, P. (2016) "Argument Mining: State of the art and emerging trends." ACM Transactions on Internet Technology, 16 (2), 2016, http://dx.doi.org/10.1145/2850417

Lytos, A., Lagkas, T., Sarigiannidis, P. e Bontcheva, K. (2019) "The evolution of argumentation mining: From models to social media and emerging tools." Information Processing \& Management, 56(6), 102055, https://doi.org/10.1016/j.ipm.2019.102055

Maas, A., Daly, R. E., Pham, P. T., Huang, D., Ng, A. Y. e Potts, C. (2011) "Learning word vectors for sentiment analysis". In Proceedings of the 49th annual meeting of the 
association for computational linguistics: Human language technologies (pp. 142150), https://www.aclweb.org/anthology/P11-1015

Nascimento, R. S., Nascimento, G., Carvalho, F. e Guedes, G. (2020). "Mineração de Opiniões com LIWC: abordagem prática sobre sistemas judiciais eletrônicos brasileiros". In Anais do IX Brazilian Workshop on Social Network Analysis and Mining (pp. 132-141). SBC, https://doi.org/10.5753/brasnam.2020.11169

NLTK (2021) “Natural Language Toolkit.”, https://www.nltk.org/

Palau, R. M. e Moens, M. F. (2009) "Argumentation mining: the detection, classification and structure of arguments in text". In Proceedings of the 12th international conference on artificial intelligence and law (pp. 98-107), https://doi.org/10.1145/1568234.1568246

Paltoglou, G. e Thelwall, M. (2012) "Twitter, MySpace, Digg: Unsupervised sentiment analysis in social media". ACM Transactions on Intelligent Systems and Technology (TIST), 3(4), 1-19, https://doi.org/10.1145/2337542.2337551

Pedregosa, F., Varoquaux, G., Gramfort, A., Michel, V., Thirion, B., Grisel, O., ... e Duchesnay, E. (2011) "Scikit-learn: Machine learning in Python". The Journal of machine Learning research, 12, 2825-2830, https://dl.acm.org/doi/10.5555/1953048.2078195

Rodrigues, A. O., de Melo-Minardi, R. C. e Junior, W. M. (2013) “Como nos Sentimos: Uma Ferramenta de Mineração Visual de Sentimentos no Twitter". In Anais do II Brazilian Workshop on Social Network Analysis and Mining (pp. 91-102). SBC, https://sol.sbc.org.br/index.php/brasnam/article/view/6836

Skoric, M. M., Zhu, Q., Goh, D. e Pang, N. (2016) "Social media and citizen engagement: A meta-analytic review". New Media \& Society, 18(9), 1817-1839. http://dx.doi.org/10.1177/1461444815616221.

Tavares, R. L, Pimentel, M., Araujo, R. M. (2012) "Visualização de Informações em Discussões Políticas". In: Brazilian Symposium on Collaborative Systems. São Paulo. Sociedade Brasileira de Computação. http://sws2012.ime.usp.br/sbsc/SBSC2012/data/4890a075.pdf

Tavares, R. L, Pimentel, M., Araujo, R. M. (2014) "Visualizing Clashes and Alliances in Social Networks of Political Discussions". Social Networking, v. 03, p. 94-101. http://dx.doi.org/10.4236/sn.2014.32012

Toulmin, S. E. (2003). "The uses of argument". Cambridge university press. https://doi.org/10.1080/00048405985200191

UFRJ. (2020) "Media Lab", https://medialabufrj.net/

Walker, M. A., Tree, J. E. F., Anand, P., Abbott, R. e King, J. (2012) “A Corpus for Research on Deliberation and Debate." In LREC (Vol. 12, pp. 812-817), https://www.aclweb.org/anthology/L12-1643/

Zhai, C. e Massung, S. (2016) "Opinion Mining and Sentiment Analysis. Text Data Management and Analysis: A Practical Introduction to Information Retrieval and Text Mining. "Association for Computing Machinery and Morgan \& Claypool, https://doi.org/10.1145/2915031.2915050 